\title{
Associations of Fibroblast Growth Factor 23 and Fetuin-A With Coronary Plaque Burden and Plaque Composition in Young Adults
}

\author{
Fatih Akin, MD, * Omer Celik, MD, † Burak Ayca, MD, Ibrahim Altun, MD, * Vesile Ornek Diker, MD, $\uparrow$ \\ Ismail Biyık, MD, † Dimitrie Siriopol, MD,\| Adrian Covic, MD,\|l and Mehmet Kanbay, MD $\mid$
}

\begin{abstract}
Objective: The total burden of subclinical coronary artery disease (CAD) is significant among young adults. Serum fibroblast growth factor 23 (FGF-23) and fetuin-A are established predictors of morbidity and mortality because of cardiovascular disease. The objective of the study was to evaluate the relationship between subclinical CAD and serum FGF-23 and fetuin-A concentrations among a population of young adults.

Methods: A total of 241 subjects younger than 45 years who had undergone coronary computed tomographic angiography (CCTA) were included in the study. In 117 patients, the CCTA detected subclinical CAD; the rest of the patients had no CAD detected on CCTA.

Results: Serum FGF-23 and fetuin-A levels were significantly increased in the CAD patients as compared with the non-CAD patients (26.7 [interquartile range, 22.4-31.9] vs 15.7 [interquartile range, $13.2-18.1$ ] pg/mL and 904.7 [interquartile range, 695.5-1021.6] vs 469.6 [331.4-660.5] $\mathrm{mg} / \mathrm{L}$, respectively; $P<0.001$ for both). Furthermore, a positive correlation was identified between FGF-23 and fetuin-A levels and the total number of plaques ( $r=0.21$ and $r=0.28$, respectively; $P<0.001$ for both). In multivariate logistic regression analysis, age, smoking status, uric acid, FGF-23, and fetuin-A levels were found to be independently associated with the presence of CAD.
\end{abstract}

Conclusions: The presence of subclinical CAD is independently associated with FGF-23 and fetuin-A and could be used as novel risk markers of cardiovascular disease in the asymptomatic young adult population.

Key Words: fibroblast growth factor 23, fetuin-A, subclinical atherosclerosis, coronary computed tomography angiography

(J Investig Med 2015;63: 613-619)

$\mathrm{F}$ ibroblast growth factor 23 (FGF-23) is a recently identified osteocyte-derived hormone that regulates phosphate and vitamin D metabolism through an established bone-kidney axis. ${ }^{1}$ Fibroblast growth factor 23 levels are higher in hemodialysis (HD) patients and have been linked to increased mortality, in that setting. ${ }^{2}$ Clear explanations for this relationship are not yet available. Emerging data are linking FGF-23 to aortic, ${ }^{3}$ peripheral vascular, ${ }^{4}$ and coronary artery ${ }^{5}$ calcifications in HD patients. Elevated serum FGF-23 levels have been found to be associated with vascular

From the *Department of Cardiology, Muğla Sttkı Kocman University School of Medicine, Muğla; †Department of Cardiology, Mehmet Akif Ersoy Chest and Cardiovascular Surgery Education and Research Hospital; and $\ddagger$ Department of Cardiology, Bağcllar Education and Research Hospital, Istanbul; and \$Department of Biochemistry, Mehmet Akif Ersoy Chest and Cardiovascular Surgery Education and Research Hospital, Istanbul, Turkey; \#Nephrology Clinic, Dialysis and Renal Transplant Center, G. I. Popa University Hospital of Medicine and Pharmacy, Iasi, Romania; and IDivision of Nephrology, Department of Medicine, Koc University School of Medicine, Istanbul, Turkey.

Received October 9, 2014, and in revised form December 13, 2014.

Accepted for publication January 1, 2015.

Reprints: Fatih Akın, MD, Orhaniye Mah, Merkez, 48000, Muğla Sitkı

Kocman University School of Medicine, Muğla, Turkey.

E-mail: faith._akin@hotmail.com, fatihakin@mu.edu.tr.

The authors have no conflicts of interest to declare.

Copyright (C) 2015 by The American Federation for Medical Research ISSN: $1081-5589$

DOI: 10.1097/JIM.0000000000000153 diseases such as carotid intima-media thickness, arterial stiffness, and coronary atherosclerosis in patients with advanced chronic kidney disease (CKD) ${ }^{6-8}$ However, the impact of FGF-23 on early stages of atherosclerosis is not known, in patients with normal kidney function. Masai et al. ${ }^{9}$ reported that serum FGF-23 levels were associated with coronary artery calcification (evaluated by Agatston score) in patients without CKD.

Fetuin-A, a known negative acute phase reactant, is a $62-\mathrm{kd}$ glycoprotein secreted by the liver that inhibits vascular calcification. ${ }^{10}$ Previous studies suggested that fetuin-A may inhibit coronary artery calcification both in patients with and without CKD. ${ }^{11,12}$ Conversely, lower plasma fetuin-A levels are associated with atherosclerosis and inflammation. ${ }^{13,14}$ Fetuin-A has been linked to diabetes mellitus in older persons. ${ }^{15}$ Fetuin-A has also been demonstrated to be a predictor of total and cardiovascular morbidity and mortality. ${ }^{14,16}$

Although the incidence of cardiovascular events is low in individuals younger than 45 years, subclinical atherosclerosis often begins in childhood or young adulthood. Early detection of coronary artery disease (CAD) in young patients is important if we want to improve the management and prognostic of this disease. Noninvasive imaging modalities, such as coronary computed tomographic angiography (CCTA), are commonly used to evaluate the presence of coronary atherosclerotic lesions. To our knowledge, there are no published data on the relationship between serum FGF-23 and fetuin-A levels and subclinical CAD detected by CCTA in young adults. Whereas FGF-23 and fetuin-A have been shown to correlate with vascular calcification, the association of FGF-23 and fetuin-A with coronary plaque morphology remains unclear. Therefore, in this study, we aimed to determine the association of FGF-23 and fetuin-A level with subclinical CAD in young patients without a history of CAD. Furthermore, we hypothesized that FGF-23 and fetuin-A would also be associated with coronary plaque burden and structure detected by CCTA in patients younger than 45 years.

\section{METHODS}

\section{Study Population}

This single-location, case-control observational investigation was performed at a tertiary heart center. A total of 241 consecutive patients younger than 45 years and who underwent CCTA between April 2011 and May 2014 were evaluated for enrolment in the study. Following initial clinical evaluation, 117 patients with $\mathrm{CAD}$ were identified. A second group of 124 subjects with normal CCTA findings served as the control group and made up the remainder of the 241 included patients in the study. Indications for CCTA evaluation were as follows: evaluation of asymptomatic patients who have intermediate- to high-risk score according to Framingham risk assessment and also who have contraindications to exercise stress test or having uninterpretable stress test results. 
The other indications were the investigation of congenital heart disease before noncoronary cardiac surgery

The criteria for exclusion were as follows: prior diagnosis of $\mathrm{CAD}$ or prior history of any coronary surgery or bypass grafting surgery, severe valve disease, the use of calcium supplements or vitamin D treatment, and uninterpretable CCTA results. All subjects provided written informed consent, demographic/clinical characteristics were recorded, and blood samples were drawn for analysis. The study protocol was reviewed and approved by an internal ethical review board.

\section{Variable Definition}

Height and body weight were recorded, and the body mass index (BMI) was calculated according to the following formula: weight in kilograms / height in meters squared. Hypertension was defined as blood pressureof $140 / 90 \mathrm{~mm} \mathrm{Hg}$ or greater or the use of antihypertensive medication. Dyslipidemia was defined as serum total cholesterol or low-density lipoprotein (LDL) cholesterol levels of $200 \mathrm{mg} / \mathrm{dL}$ or greater and $110 \mathrm{mg} / \mathrm{dL}$, respectively, or the use of hypolipidemic medication.

\section{Image Analysis}

All scans were evaluated using the 3-dimensional Syngo.via workstation (Siemens Healthcare, Forchheim, Germany) by a pair of highly trained radiologists who were unaware of the clinical reports. The final CCTA diagnosis was determined by consensus interpretation. The identification of plaque and stenosis, as well as the amount of plaques per segment, was determined by the radiologists as indicated by the modified American Heart Association classification. ${ }^{17}$

Plaques were defined as structures in the artery that were within $1 \mathrm{~mm}^{2}$ or the vessel lumen or directly adjacent and could be clearly distinguished from the surrounding pericardial tissue and the vessel lumen. Calcified plaques were made up of more than $50 \%$ calcified tissue (density $\mathrm{C} 130 \mathrm{HU}$ in native scans); mixed plaques contained less than $50 \%$ calcified tissue; plaques lacking signs of calcification were categorized as noncalcified lesions. ${ }^{18}$ Subclinical CAD was defined as the presence of plaque during the CCTA examination in an asymptomatic patient.

\section{Laboratory Measurements}

Venous blood was collected from all study subjects after an overnight fast between 8:00 and 9:00 AM at the beginning of the study. An Abbott Aeroset autoanalyzer (Abbott Laboratories, Abbott Park, IL) was used to measure triglycerides, high-density lipoprotein cholesterol (HDL-C), and total cholesterol, in blood serum samples. The Friedenwald equation was used to calculate LDL-C levels. An immunoturbidimetric method (Abbott Aeroset 1600 autoanalyzer; Abbott Reagents, Wiesbaden, Germany) was used to determine the high-sensitivity C-reactive protein (hs-CRP) concentration. Hemoglobin and other hematologic parameters were measured on Cell-Dyne counter of Abbott Laboratories (Celldyne 3700; Abbott Laboratories). Creatinine was measured by a qualified technician using enzymatic assays (Cobas 6000; Roche, Mannheim, Germany). Blood urea nitrogen (BUN) levels were calculated from the decrease in NADH absorbance at $340 \mathrm{~nm}$ using a BUN Sigma standard. The assessment of renal function was based on the MDRD eGFR equation. ${ }^{19}$ Serum concentrations of FGF-23 and fetuin-A were measured in BD Vacutainer serum tube stored at $-40^{\circ} \mathrm{C}$ until testing. An enzymatic colorimetric test was used to measure uric acid levels on a Roche/ Hitachi analyzer (Cobas 6000; Roche). Fibroblast growth factor 23 was measured using an enzyme-linked immunosorbent assay kit from EMD Millipore Corporation (Billerica, MA). The intra-assay and interassay coefficients of variation were $9.3 \%$ and $5.08 \%$, respectively, for the FGF-23. Fetuin-A (AssayMax Human alpha-2-HS-Glycoprotein-AHSG-ELISA Kit; Assaypro, St Charles, MO) were analyzed by the enzyme-linked immunosorbent assay method. The intra-assay and interassay coefficients of variation were $5.0 \%$ and $7.0 \%$, respectively, for the fetuin-A.

\section{Statistical Analysis}

All analyses were performed using SPSS 20.0 (released 2011, IBM statistics for Windows version 20, IBM Corp, Armonk, NY). Continuous data are presented as median with interquartile range (IR), and categorical data as number and percent frequency. Comparisons between groups were performed by Mann-Whitney $U$ or $\chi^{2}$ test. Spearman or point-biserial correlation coefficient was computed to examine the association between variables. Logistic regression analysis was used to assess predictors of subclinical CAD. Those variables with $P<0.1$ by univariate analysis were included in the backward stepwise multivariate logistic regression analysis model, and the respective odds ratios (ORs) with 95\% confidence intervals (CIs) were calculated. A receiver operating characteristic curve was constructed to determine FGF-23 and fetuin-A cutoff values for the diagnosis of the subclinical coronary atherosclerosis. When a significant cutoff value was observed, the sensitivity and specificity values were presented. A 2 -tailed $P<0.05$ was considered statistically significant.

\section{RESULTS}

\section{Study Population Characteristics}

The clinical, biological, and demographic characteristics of the study population are presented in Table 1 . The median age was 42 years, and $59.8 \%$ were men. Patients with CAD were older and had a higher prevalence of hypertension and of tobacco use. Similarly, serum creatinine, hs-CRP, uric acid, LDL-C, and triglycerides values were more elevated in the $\mathrm{CAD}$ patients as compared with those without CAD. Importantly, both FGF-23 and fetuin-A levels were also significantly higher in the CAD group of patients (26.7 [IR, 22.4-31.9] pg/mL vs 15.7 [IR, 13.2-18.1] $\mathrm{pg} / \mathrm{mL}$ and 904.7 [IR, 695.5-1021.6] $\mathrm{mg} / \mathrm{L}$ vs 469.6 [IR, $331.4-660.5] \mathrm{mg} / \mathrm{L}$, respectively; $P<0.001$ for both). There was no difference between the 2 groups in regard to diabetes status and hemoglobin $\mathrm{A}_{1 \mathrm{c}}$, calcium, or phosphorus levels.

In the CAD group, the median number of vessels affected was 2 (IR, 1-3), and the most frequent type of plaque present was the mixed one $(49.6 \%)$, followed by the noncalcified $(39.3 \%)$ and calcified (11.1\%) types. The fetuin-A and FGF-23 values were similar between patients with less than 2 and those with 2 or more vessels affected. There was no difference in the fetuin-A levels between the patients with different types of plaques, but patients with noncalcified plaques had significantly higher values of FGF-23 than did those with calcified plaques (27.6 [IR, 24.5-34.6] pg/ $\mathrm{mL}$ vs 23.9 [IR, 20.8-27.0] pg/mL, $P=0.01$; Fig. 1).

\section{Association Between FGF-23, Fetuin-A, and Different Parameters}

Fibroblast growth factor 23 and fetuin-A showed a positive correlation $(r=0.42, P<0.001)$. Both biomarkers were associated with age, systolic blood pressure, uric acid, and hs-CRP levels, different parameters of lipid metabolism and, importantly, with the presence of CAD $(r=0.21$ for FGF-23 and $r=0.28$ for fetuin-A, respectively; $P<0.001$ ), number of plaques, and of 
TABLE 1. Characteristics of the Study Population

\begin{tabular}{|c|c|c|c|c|}
\hline & All $(n=241)$ & Non-CAD $(n=124)$ & $\operatorname{CAD}(n=117)$ & $\boldsymbol{P}$ \\
\hline Age, y & $42.0(39.0-44.0)$ & $40.0(38.0-43.0)$ & $42.0(40.5-44.0)$ & $<0.001$ \\
\hline Male gender, n (\%) & $144(59.8)$ & $67(54.0)$ & $77(65.8)$ & 0.07 \\
\hline Hypertension, n (\%) & $61(25.3)$ & $18(14.5)$ & $43(36.8)$ & $<0.001$ \\
\hline SBP, mm Hg & $125(115-135)$ & $120(110-130)$ & $130(120-135)$ & $<0.001$ \\
\hline DBP, mm Hg & $80(70-85)$ & $75(70-80)$ & $80(70-85)$ & 0.01 \\
\hline Diabetes mellitus, n (\%) & $57(23.7)$ & $27(21.8)$ & $30(25.6)$ & 0.48 \\
\hline Family history, n (\%) & $109(45.2)$ & $59(47.6)$ & $50(42.7)$ & 0.45 \\
\hline Smoking, n (\%) & $113(46.9)$ & $30(24.2)$ & $83(70.9)$ & $<0.001$ \\
\hline BMI, $\mathrm{kg} / \mathrm{m}^{2}$ & $28.3(26.8-30.4)$ & $28.1(26.6-29.8)$ & $28.4(26.8-31.1)$ & 0.09 \\
\hline Hemoglobin (12.5-16), g/dL & $14.0(12.9-15.0)$ & $13.6(12.5-14.7)$ & $14.0(13.0-15.0)$ & 0.001 \\
\hline White blood cell, $\times 10^{3} / \mu \mathrm{L}$ & $7.6(6.4-8.9)$ & $7.5(6.4-8.8)$ & $7.7(6.5-9.3)$ & 0.35 \\
\hline Platelet $(130-400), \times 10^{3} / \mu \mathrm{L}$ & $264.0(228.0-306.5)$ & $261.0(222.0-303.8)$ & $269.0(242.0-315.0)$ & 0.06 \\
\hline $\mathrm{BUN}, \mathrm{mg} / \mathrm{dL}$ & $12.0(10.0-12.0)$ & $12.0(10.0-12.3)$ & $12.0(10.0-12.0)$ & 0.65 \\
\hline Creatinine $(0.4-0.9), \mathrm{mg} / \mathrm{dL}$ & $0.7(0.6-0.8)$ & $0.7(0.6-0.8)$ & $0.7(0.6-0.9)$ & 0.76 \\
\hline $\mathrm{eGFR}, \mathrm{mL} / \mathrm{min}$ per $1.73 \mathrm{~m}^{2}$ & $117.0(99.0-147.0)$ & $118.0(98.3-147.8)$ & $115.0(100.0-144.5)$ & 0.61 \\
\hline Uric acid (2.4-5.7), mg/dL & $5.1(4.4-6.1)$ & $4.8(4.0-5.3)$ & $5.7(4.7-6.8)$ & $<0.001$ \\
\hline Total cholesterol, mg/dL & $198.0(176.0-230.0)$ & $192.0(173.0-216.0)$ & $212.0(181.5-242.0)$ & $<0.001$ \\
\hline $\operatorname{LDL}(0-130), \mathrm{mg} / \mathrm{dL}$ & $126.0(109.2-155.5)$ & $123.3(103.0-138.0)$ & $136.0(112.0-166.0)$ & $<0.001$ \\
\hline HDL (45-65), mg/dL & $40.0(34.0-46.0)$ & $43.0(35.3-49.5)$ & $38.0(31.0-44.0)$ & $<0.001$ \\
\hline Triglycerides $(0-200), \mathrm{mg} / \mathrm{dL}$ & $142.0(99.5-192.5)$ & $120.5(94.0-163.3)$ & $153.0(110.0-230.0)$ & $<0.001$ \\
\hline $\mathrm{HbA}_{1 \mathrm{c}}, \%$ & $5.7(5.6-6.0)$ & $5.8(5.6-6.1)$ & $5.7(5.6-5.9)$ & 0.60 \\
\hline hs-CRP $(0-5), \mathrm{mg} / \mathrm{L}$ & $1.3(1.0-2.1)$ & $1.2(0.9-1.5)$ & $1.8(1.3-2.2)$ & $<0.001$ \\
\hline Calcium (8.8-10.2), mg/dL & $9.3(8.6-9.9)$ & $9.3(8.5-9.9)$ & $9.3(8.6-9.9)$ & 0.30 \\
\hline Phosphorus (2.5-4.5), mg/dL & $3.3(3.1-3.6)$ & $3.3(3.1-3.6)$ & $3.3(3.1-3.7)$ & 0.98 \\
\hline FGF-23, pg/mL & $20.0(15.4-26.8)$ & $15.7(13.2-18.1)$ & $26.7(22.4-31.9)$ & $<0.001$ \\
\hline Fetuin-A, mg/L & $675.8(449.5-944.7)$ & $469.6(331.4-660.5)$ & $904.7(695.5-1021.6)$ & $<0.001$ \\
\hline
\end{tabular}

DBP indicates diastolic blood pressure; eGFR, estimated glomerular filtration rate; $\mathrm{HbA}_{1 \mathrm{c}}$, hemoglobin $\mathrm{A}_{1 \mathrm{c}} ; \mathrm{SBP}$, systolic blood pressure.

Data are expressed as median with interquartile range or number with percent frequency, as appropriate.

vessels affected (Table 2, Fig. 2). There was no association with calcium and phosphorus levels.

\section{Predictors of CAD}

Next, we analyzed the predictors of the presence CAD in the studied population. Table 3 shows the univariate predictors of CAD identified by logistic regression. In this analysis, both FGF-23 and fetuin-A were predictors of CAD. After the inclusion of all significant variables and also gender $(P=0.06$ in univariate analysis) in a multivariable logistic regression, these biomarkers remained independently associated with the presence of CAD (Table 4).

When we compared the area under the curve (AUC) of the receiver operating characteristic curve for the FGF-23 and fetuin-A, FGF-23 had a significantly larger AUC than that of fetuin-A (AUC, 0.924 [95\% CI, 0.887-0.961] vs 827 [95\%
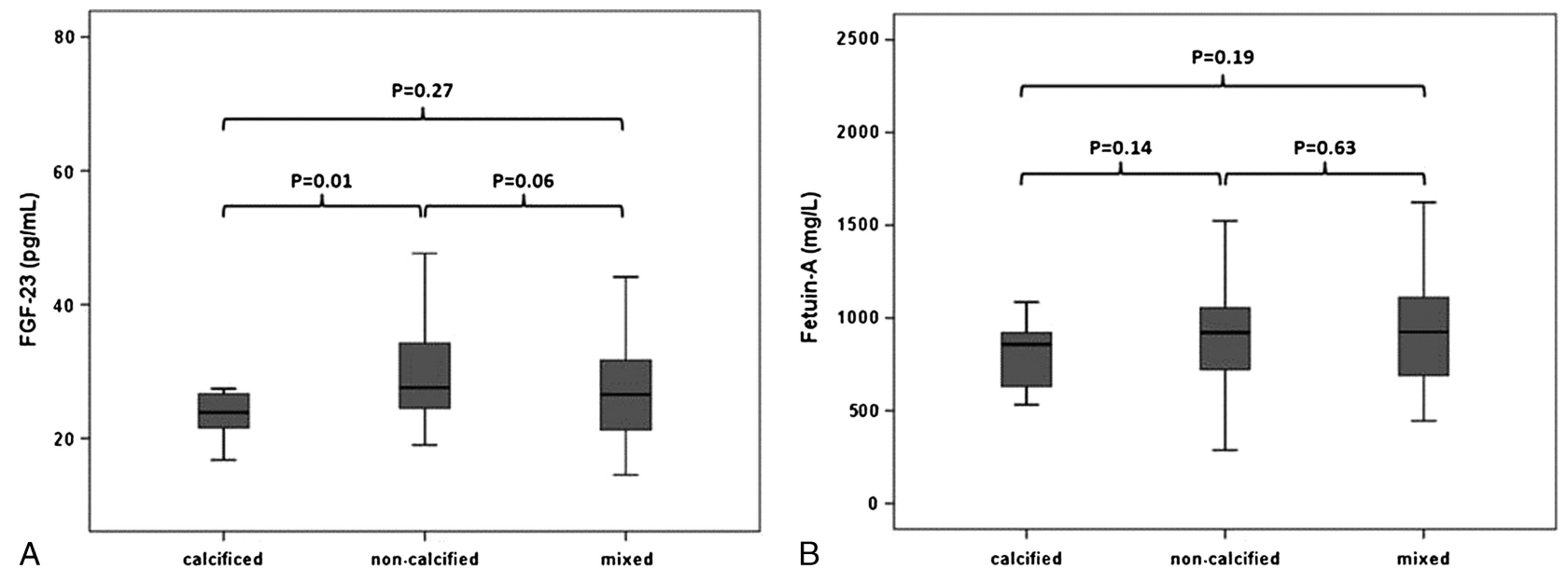

FIGURE 1. Fibroblast growth factor 23 (in pg/mL) (A) and fetuin-A (in $\mathrm{mg} / \mathrm{L}$ ) (B) across the different plaque types. 
TABLE 2. Correlations Between FGF-23 and Fetuin-A Levels and different Variables Evaluated in the Study

\begin{tabular}{|c|c|c|c|c|}
\hline & \multicolumn{2}{|c|}{ FGF-23 } & \multicolumn{2}{|c|}{ Fetuin-A } \\
\hline & Correlation Coefficient & $\boldsymbol{P}$ & Correlation Coefficient & $\boldsymbol{P}$ \\
\hline Age, y & 0.19 & 0.03 & 0.16 & $<0.001$ \\
\hline Gender & 0.02 & 0.75 & 0.13 & 0.05 \\
\hline Hypertension & 0.13 & 0.04 & 0.09 & 0.14 \\
\hline SBP, mm Hg & 0.20 & 0.01 & 0.17 & 0.01 \\
\hline DBP, mm Hg & 0.11 & 0.08 & 0.17 & 0.01 \\
\hline Diabetes mellitus & -0.02 & 0.74 & -0.01 & 0.99 \\
\hline Family history & -0.06 & 0.38 & -0.02 & 0.79 \\
\hline Smoking & 0.09 & 0.16 & 0.21 & $<0.001$ \\
\hline BMI, $\mathrm{kg} / \mathrm{m}^{2}$ & 0.03 & 0.68 & 0.13 & 0.51 \\
\hline Hemoglobin, g/dL & 0.16 & 0.01 & 0.13 & 0.04 \\
\hline White blood cell, $\times 10^{3} / \mu \mathrm{L}$ & 0.06 & 0.34 & 0.14 & 0.04 \\
\hline Platelet, $\times 10^{3} / \mu \mathrm{L}$ & 0.14 & 0.03 & 0.07 & 0.28 \\
\hline BUN, mg/dL & -0.09 & 0.13 & -0.04 & 0.56 \\
\hline Creatinine, $\mathrm{mg} / \mathrm{dL}$ & 0.12 & 0.07 & 0.19 & 0.04 \\
\hline $\mathrm{eGFR}, \mathrm{mL} / \mathrm{min}$ per $1.73 \mathrm{~m}^{2}$ & 0.02 & 0.72 & -0.06 & 0.39 \\
\hline Uric acid, mg/dL & 0.21 & $<0.001$ & 0.33 & $<0.001$ \\
\hline Total cholesterol, mg/dL & 0.17 & 0.01 & 0.14 & 0.03 \\
\hline $\mathrm{LDL}, \mathrm{mg} / \mathrm{dL}$ & 0.13 & 0.04 & 0.09 & 0.14 \\
\hline $\mathrm{HDL}, \mathrm{mg} / \mathrm{dL}$ & -0.24 & $<0.001$ & -0.11 & 0.09 \\
\hline Triglycerides, mg/dL & 0.18 & 0.01 & 0.19 & 0.01 \\
\hline $\mathrm{HbA}_{1 \mathrm{c}}, \%$ & 0.03 & 0.63 & -0.08 & 0.22 \\
\hline Hs-CRP, mg/L & 0.25 & $<0.001$ & 0.19 & 0.01 \\
\hline Calcium, mg/dL & 0.05 & 0.44 & 0.01 & 0.86 \\
\hline Phosphorus, mg/dL & 0.02 & 0.75 & 0.03 & 0.69 \\
\hline CAD & 0.31 & $<0.001$ & 0.42 & $<0.001$ \\
\hline No. plaques & 0.21 & 0.001 & 0.28 & $<0.001$ \\
\hline No. vessels affected & 0.69 & $<0.001$ & 0.54 & $<0.001$ \\
\hline
\end{tabular}

DBP, diastolic blood pressure; eGFR, estimated glomerular filtration rate; $\mathrm{HbA}_{1 c}$, hemoglobin $\mathrm{A}_{1 \mathrm{c}} ; \mathrm{SBP}$, systolic blood pressure.

Boldface indicates values are statistically significant.

CI, 0.773-0.872]; $P<0.01)$ for the prediction of coronary atherosclerosis (Fig. 3). We also identified a cutoff for both biomarkers for the diagnosis of CAD: for FGF-23 $18.61 \mathrm{pg} / \mathrm{mL}$ (sensitivity $95.73 \%$, specificity $82.26 \%$ ) and for fetuin-A $573 \mathrm{mg} / \mathrm{L}$ (sensitivity $91.45 \%$, specificity $65.32 \%$ ).

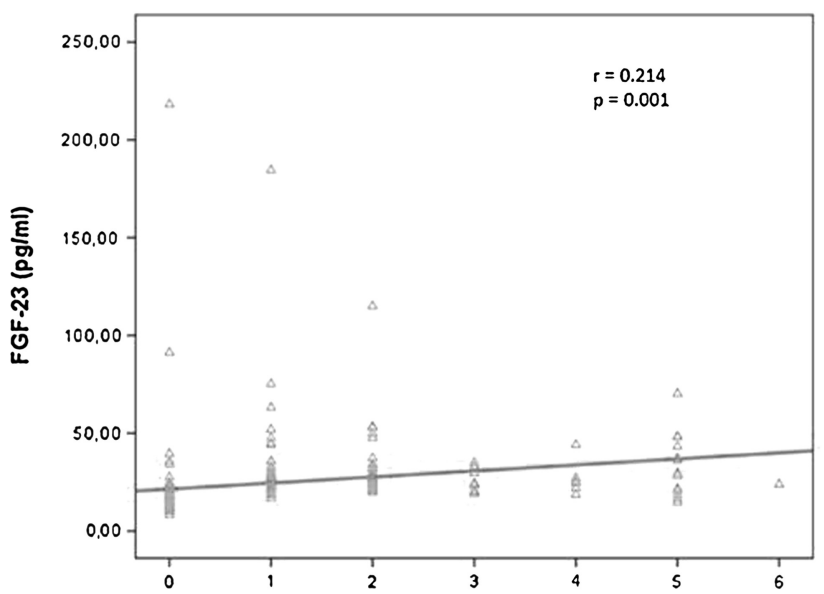

A number of plaques

\section{DISCUSSION}

In the present study, we show for the first time that FGF-23 and fetuin-A levels are independently associated with subclinical $\mathrm{CAD}$ among asymptomatic young adults. Fibroblast growth factor 23 and fetuin-A values were also positively correlated with

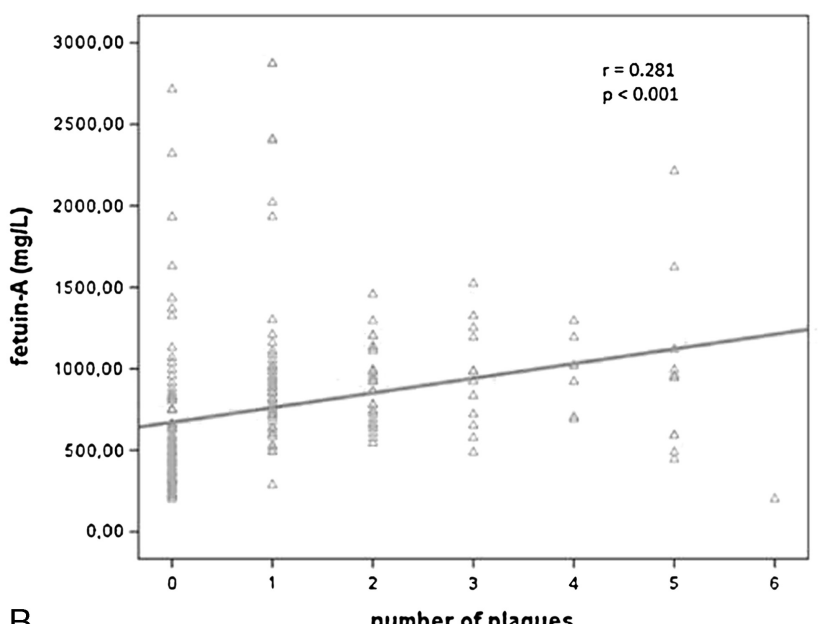

B

FIGURE 2. Correlation between FGF-23 and number of plaques (A), and correlation between fetuin-A and number of plaques (B). 
TABLE 3. Univariate Logistic Regression for Factors Associated With CAD

\begin{tabular}{lccc}
\hline & OR & 95\% CI & $\boldsymbol{P}$ \\
\hline Age, y & 1.165 & $1.080-1.255$ & $<0.001$ \\
Hypertension $(0=$ no, $1=$ yes $)$ & 3.422 & $1.831-6.394$ & $<0.001$ \\
Smoking $(0=$ no, $1=$ yes $)$ & 7.649 & $4.313-13.564$ & $<0.001$ \\
BMI, kg/m ${ }^{2}$ & 1.097 & $1.026-1.173$ & 0.01 \\
Hemoglobin, g/dL & 1.348 & $1.130-1.608$ & 0.001 \\
Creatinine, mg/dL & 11.159 & $2.519-49.440$ & 0.001 \\
Uric acid, mg/dL & 2.125 & $1.650-2.737$ & $<0.001$ \\
Dyslipidemia $(0=$ no, $1=$ yes $)$ & 2.197 & $1.313-3.678$ & 0.01 \\
Hs-CRP, mg/L & 1.403 & $1.070-1.840$ & 0.01 \\
FGF-23, pg/mL & 1.123 & $1.078-1.170$ & $<0.001$ \\
Fetuin-A, mg/L & 1.003 & $1.002-1.004$ & $<0.001$ \\
\hline
\end{tabular}

the number of affected vessels, as well as with the number of plaques in the coronary arteries.

Atherosclerosis is the largest cause of morbidity and mortality in the developed world. Its pathogenesis can begin early in life and occurs in a substantial number of asymptomatic adults. ${ }^{20}$ The relation between FGF-23, fetuin-A, and CAD has been demonstrated previously by coronary angiography. ${ }^{8,21}$ In our study, CAD was demonstrated by CCTA, which provides more accurate and reliable information about early stage atherosclerosis. Furthermore, CCTA also offers additional information as compared with coronary angiography, being able to provide, with high diagnostic accuracy, details about plaque composition.

Fibroblast growth factor 23 may play a direct role in vascular pathology, although the mechanism remains unclear. It was recently demonstrated that Klotho, which determines the tissue-specific signaling of FGF-23, is expressed in human arteries and human aortic smooth muscle cells, ${ }^{22}$ suggesting that FGF-23 could affect vascular tissues in an endocrine fashion. ${ }^{23}$ Fibroblast growth factor 23 may also affect the cardiovascular system indirectly through regulation of mineral metabolism. Fibroblast growth factor 23 regulates systemic phosphate homeostasis, and there is a significant association between atherosclerosis and phosphate metabolism in patients with $\mathrm{CKD}^{24}$; however, in our non-CKD population, serum phosphate did not have an influence on the relationship between FGF23 and CAD. Fibroblast growth factor 23 may also modulate progression and development of coronary atherosclerosis through its inhibition of vitamin D activation. ${ }^{25}$

The association of fetuin-A with atherosclerosis is controversial. Several studies have shown a negative relationship between fetuin-A and subclinical atherosclerosis in patients with endstage renal disease and obstructive sleep apnea syndrome. ${ }^{26,27}$ By contrast, and similar to our findings, fetuin-A has been found to be positively correlated with carotid artery intima-media thickness in patients with nonalcoholic fatty liver disease ${ }^{28}$ or in

TABLE 4. Backward Stepwise Multivariable Logistic Regression for Factors Associated With CAD

\begin{tabular}{lccc}
\hline & OR & $\mathbf{9 5 \%}$ CI & $\boldsymbol{P}$ \\
\hline Age, y & 1.173 & $1.062-1.296$ & 0.01 \\
Smoking $(0=$ no, 1 = yes $)$ & 6.544 & $3.111-13.762$ & $<0.001$ \\
Uric acid, mg/dL & 2.039 & $1.461-2.845$ & $<0.001$ \\
FGF-23 & 1.049 & $1.013-1.087$ & 0.01 \\
Fetuin-A & 1.002 & $1.001-1.003$ & $<0.001$ \\
\hline
\end{tabular}

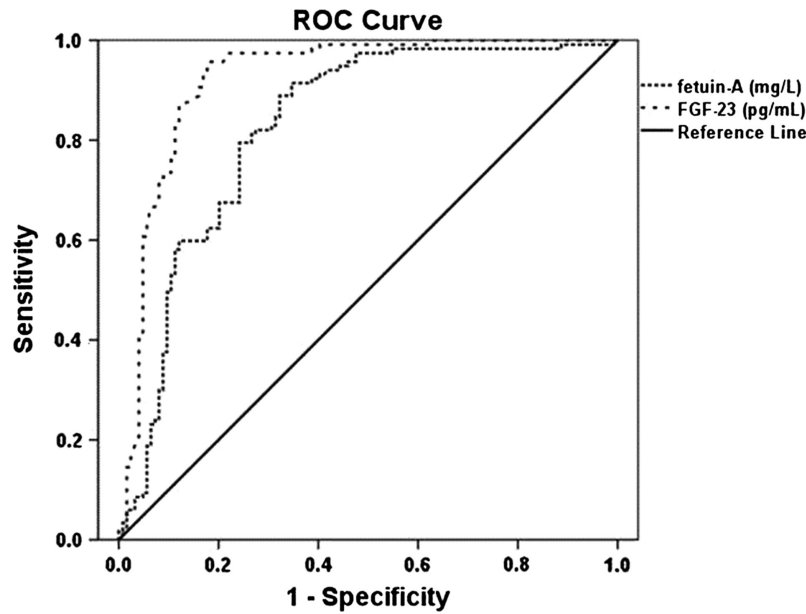

FIGURE 3. Receiver operating characteristic curve for serum FGF-23 and fetuin-A as a predictors of coronary atherosclerosis.

patients with normal renal function. ${ }^{29}$ Rittig et al. ${ }^{13}$ reported that serum fetuin-A levels were higher in patients with subclinical atherosclerosis than in healthy subjects, in a middle-aged population. It was also reported that higher plasma fetuin-A levels were associated with risk of both myocardial infarction and ischemic stroke in middle-aged subjects. ${ }^{30}$ In the Rancho Bernardo study, ${ }^{16}$ lower fetuin-A levels were significantly associated with increased risk of cardiovascular mortality in older adults and with reduced risk of cardiovascular mortality in younger and diabetic adults. These findings imply that the negative effects associated with fetuin-A levels could differ with age, with higher levels being protective in older patients or in those who have an elderly-like phenotype (as in end-stage renal disease) and being deleterious in younger patients.

There are conflicting data on the association between fetuin-A levels and vascular calcification. Several studies have shown a negative relationship between fetuin-A and vascular calcification in patients with end-stage renal disease. ${ }^{11,14}$ By contrast, Mehrotra et al. ${ }^{31}$ found a positive correlation between fetuin-A and coronary artery calcification.

Fetuin-A is known to be a negative acute phase reactant, and its associations with subclinical atherosclerosis might be influenced by the presence of inflammation. A direct link between fetuin-A deficiency and CRP has been described in patients on HD. ${ }^{14}$ Although we found a positive relationship between fetuin-A and CRP levels, only fetuin-A was independently associated with $\mathrm{CAD}$ in our multivariable logistic regression. Fetuin-A may be related to the increased peripheral tissue resistance to insulin, as it was reported that fetuin-A may inhibit the autophosphorylation of the insulin receptor. ${ }^{32,33}$ In line with this assumption, fetuin-A was shown to be correlated with diabetes and hyperlipidemia, ${ }^{34}$ known risk factors for the development of atherosclerotic lesions. However, in the present study, fetuin-A was not significantly associated with diabetes and, although it was correlated with total cholesterol and triglycerides levels, still remained positively associated with CAD after multiple adjustments for dyslipidemia markers.

A final mechanism proposed to explain the relation between FGF-23 and fetuin-A with subclinical atherosclerosis may be the increased risk of coronary calcification. Calcification is the common anatomopathologic change of subclinical CAD and is strongly associated with cardiovascular mortality. A growing body of evidence from experimental, clinical, and epidemiological studies suggests a possible association between fetuin-A, FGF-23, and vascular calcification in patients with end-stage renal disease. ${ }^{5,35}$ However, in a 
study with a small sample size that included only patients with normal renal function, both FGF-23 and fetuin-A have not been found to be correlated with the coronary artery calcification score, the grade of coronary artery stenosis, or presence of noncalcified plaques. ${ }^{36}$ Voigt et al. ${ }^{37}$ investigated FGF-23 and fetuin-A expression by means of immunohistochemistry in human atherosclerotic vessels derived from patients with preserved renal function. In their study, expression of fetuin-A was seen within areas of vascular smooth muscle actin-positive cells adjacent to calcific deposits, and serum FGF-23 levels were positively correlated with the severity of carotid atheroma calcification. Our study confirms and extends these findings. With the exception of significantly higher FGF-23 levels in the patients with noncalcified plaques as compared with those with calcified ones, we did not observe a difference in the FGF-23 or fetuin-A levels between the different plaque types.

There are some limitations in the present study. First, the sample size in our study was relatively small. Second, the prognostic values of FGF-23 and fetuin-A levels were not evaluated in a follow-up study. Third, vitamin D was not measured in our population. Fibroblast growth factor 23 might have an effect on coronary atherosclerosis indirectly through its inhibition of vitamin D activation. Finally, results may not be applicable to other ethnic groups or populations. However, whereas previous studies have used carotid intima-media thickness and coronary artery calcium score to define subclinical atherosclerosis, this is the first study that uses CCTA with an adequate analysis of coronary vessel and plaque composition to demonstrate an association between CAD and serum FGF-23 and fetuin-A levels in young adults.

In conclusion, FGF-23 and fetuin-A are independent predictors of the presence of subclinical CAD. In addition, serum FGF23 and fetuin-A were correlated with the total number of plaques and with the number of involved arteries. Finally, FGF-23 and fetuin-A could be used as novel risk markers of cardiovascular disease in non-CKD patients.

\section{REFERENCES}

1. Shimada T, Hasegawa H, Yamazaki Y, et al. FGF-23 is a potent regulator of vitamin D metabolism and phosphate homeostasis. $J$ Bone Miner Res. 2004;19:429-435

2. Gutiérrez OM, Januzzi JL, Isakova T, et al. Fibroblast growth factor 23 and mortality among patients undergoing hemodialysis. $N$ Engl J Med 2008;359:584-592.

3. Nasrallah MM, El-Shehaby AR, Salem MM, et al. Fibroblast growth factor-23 (FGF-23) is independently correlated to aortic calcification in haemodialysis patients. Nephrol Dial Transplant. 2010;25: 2679-2685.

4. Jean G, Bresson E, Terrat JC, et al. Peripheral vascular calcification in long-haemodialysis patients: associated factors and survival consequences. Nephrol Dial Transplant. 2009;24:948-955.

5. Gutiérrez OM, Januzzi JL, Isakova T, et al. Fibroblast growth factor 23 and left ventricular hypertrophy in chronic kidney disease. Circulation. 2009;119:2545-2552.

6. Balci M, Kirkpantur A, Gulbay M, et al. Plasma fibroblast growth factor-23 levels are independently associated with carotid artery atherosclerosis in maintenance hemodialysis patients. Hemodial Int. 2010;14:425e32.

7. Mirza MA, Larsson A, Lind L, et al. Circulating fibroblast growth factor-23 is associated with vascular dysfunction in the community. Atherosclerosis. 2009;205:385-390.

8. Kanbay M, Nicoleta M, Selcoki Y, et al. Fibroblast growth factor 23 and fetuin $\mathrm{A}$ are independent predictors for the coronary artery disease extent in mild chronic kidney disease. Clin J Am Soc Nephrol. 2010; 5:1780e6
9. Masai H, Joki N, Sugi K, et al. A preliminary study of the potential role of FGF-23 in coronary calcification in patients with suspected coronary artery disease. Atherosclerosis. 2013;226:228-233.

10. Lebreton JP, Joisel F, Raoult JP, et al. Serum concentration of human alpha 2 HS glycoprotein during the inflammatory process: evidence that alpha 2 HS glycoprotein is a negative acute-phase reactant. J Clin Invest. 1979;64:1118-1129.

11. Cozzolino M, Galassi A, Biondi ML, et al. Serum fetuin-A levels link inflammation and cardiovascular calcification in hemodialysis patients. Am J Nephrol. 2006;26:423-429.

12. Mori K1, Ikari Y, Jono S, et al. Fetuin-A is associated with calcified coronary artery disease. Coron Artery Dis. 2010;21:281-285.

13. Rittig K, Thamer C, Haupt A, et al. High plasma fetuin-A is associated with increased carotid intima-media thickness in a middle-aged population. Atherosclerosis. 2009;207(2):341-342.

14. Ketteler M, Bongartz P, Westenfeld R, et al. Association of low fetuin-A (AHSG) concentrations in serum with cardiovascular mortality in patients on dialysis: a cross-sectional study. Lancet. 2003;361:827-833.

15. Ix JH, Biggs ML, Mukamal KJ, et al. Association of fetuin-a with incident diabetes mellitus in community-living older adults: the cardiovascular health study. Circulation. 2012;125(19):2316-2322.

16. Laughlin GA, Cummins KM, Wassel CL, et al. The association of fetuin-A with cardiovascular disease mortality in older community-dwelling adults: the Rancho Bernardo study. J Am Coll Cardiol. 2012;59:1688-1696.

17. Austen WG, Edwards JE, Frye RL, et al. A reporting system on patients evaluated for coronary artery disease. Report of the Ad Hoc Committee for Grading of Coronary Artery Disease, Council on Cardiovascular Surgery, American Heart Association. Circulation. 1975;51:5-40.

18. Leber AW, Becker A, Knez A, et al. Accuracy of 64-slice computed tomography to classify and quantify plaque volumes in the proximal coronary system: a comparative study using intravascular ultrasound. $\mathrm{J} \mathrm{Am}$ Coll Cardiol. 2006;47:672-677.

19. Levey AS, Greene T, Beck GJ, et al. Dietary protein restriction and the progression of chronic renal disease: what have all of the results of the MDRD study shown? J Am Soc Nephrol. 1999;10:2426-2439.

20. Tuzcu EM, Kapadia SR, Tutar E, et al. High prevalence of coronary atherosclerosis in asymptomatic teenagers and young adults: evidence from intravascular ultrasound. Circulation. 2001;103:2705-2710.

21. Chen YC, Lin FY, Lin RH, et al. Relation between fetuin-A levels and fibroblast growth factor 23 with the severity of coronary artery disease measured by SYNTAX scores. Am J Cardiol. 2013;112:950-953.

22. Donate-Correa J, Mora-Fernández C, Martínez-Sanz R, et al. Expression of FGF23/KLOTHO system in human vascular tissue. Int J Cardiol. 2013, 165:179-183.

23. Lim K, Lu TS, Molostvov G, et al. Vascular Klotho deficiency potentiates the development of human artery calcification and mediates resistance to FGF-23. Circulation. 2012;125:2243-2255

24. Gutierrez O, Isakova T, Rhee E, et al. Fibroblast growth factor-23 mitigates hyperphosphatemia but accentuates calcitriol deficiency in chronic kidney disease. J Am Soc Nephrol. 2005;16:2205-2215.

25. Tuñón J, Cristóbal C, Tarín N, et al. Coexistence of low vitamin D and high fibroblast growth factor-23 plasma levels predicts an adverse outcome in patients with coronary artery disease. PLoS One. 2014;9:e95402.

26. Pertosa G, Simone S, Ciccone M, et al. Serum fetuin a in hemodialysis: a link between derangement of calcium-phosphorus homeostasis and progression of atherosclerosis? Am J Kidney Dis. 2009;53: $467-474$

27. Akyuz A, Oran M, Alpsoy S, et al. Association between serum fetuin-A levels, carotid artery stiffness, and intima-media thickness in patients with normotensive obstructive sleep apnea syndrome. Angiology. 2013;65: $607-613$ 
28. Dogru T, Genc H, Tapan S, et al. Plasma fetuin-A is associated with endothelial dysfunction and subclinical atherosclerosis in subjects with nonalcoholic fatty liver disease. Clin Endocrinol (Oxf). 2013;78:712-717.

29. Fiore CE, Celotta G, Politi GG, et al. Association of high alpha2-Heremans-Schmid glycoprotein/fetuin concentration in serum and intima-media thickness in patients with atherosclerotic vascular disease and low bone mass. Atherosclerosis. 2007;195:110-115.

30. Weikert C, Stefan N, Schulze MB, et al. Plasma fetuin-a levels and the risk of myocardial infarction and ischemic stroke. Circulation. 2008;118: 2555-2562.

31. Mehrotra R, Westenfeld R, Christenson P, et al. Serum fetuin-A in nondialyzed patients with diabetic nephropathy: relationship with coronary artery calcification. Kidney Int. 2005;67:1070-1077.

32. Auberger P, Falquerho L, Contreres JO, et al. Characterization of a natural inhibitor of the insulin receptor tyrosine kinase: cDNA cloning, purification, and anti-mitogenic activity. Cell. 1989;58:631-640.
33. Srinivas PR, Wagner AS, Reddy LV, et al. Serum alpha 2-HS-glycoprotein is an inhibitor of the human insulin receptor at the tyrosine kinase level. Mol Endocrinol. 1993;7:1445-1455.

34. Xu Y, Xu M, Bi Y, et al. Serum fetuin-A is correlated with metabolic syndrome in middle-aged and elderly Chinese. Atherosclerosis. 2011;216: 180-186

35. Coen G, De Paolis P, Ballanti P, et al. Peripheral artery calcifications evaluated by histology correlate to those detected by CT: relationships with fetuin-A and FGF-23. J Nephrol. 2011;24:313-321.

36. Roos M, Lutz J, Salmhofer H, et al. Relation between plasma fibroblast growth factor-23, serum fetuin-A levels and coronary artery calcification evaluated by multislice computed tomography in patients with normal kidney function. Clin Endocrinol (Oxf). 2008;68:660-665.

37. Voigt M, Fischer DC, Rimpau M, et al. Fibroblast growth factor (FGF)-23 and fetuin-A in calcified carotid atheroma. Histopathology. 2010;56(6): 775-788. 